\title{
Range-Relaxed Graceful Game on Graphs
}

\section{Deise L. de Oliveira, Simone Dantas, ${ }^{\text {At }}$ Atílio G. Luiz}

\begin{abstract}
Given a graph $G=(V, E)$ and a set of integer labels $\mathcal{L}=$ $\{0, \ldots, k\}, k \geq|E(G)|$, a range-relaxed graceful labeling is a function $f: V(G) \rightarrow \mathcal{L}$ such that: $(i) f$ is injective; $(i i)$ the absolute difference of the labels of the endpoints of an edge $u v \in E(G)$ already labeled is unique. The range-relaxed graceful game ( $\mathrm{RRG}$ game) is a graph labeling game recently introduced by Tuza and it is a variant of a two person graph labeling game. The players, traditionally called Alice and Bob, alternately assign an unused label $f(v) \in \mathcal{L}=\{0, \ldots, k\}$, $k \geq|E(G)|$, to an unlabeled vertex $v \in V(G)$ and the induced edge label is given by $\phi(u v)=|f(u)-f(v)|$. Alice's goal is to end up with an injective vertex labeling of all vertices of $G$ which induces distinct edge labels, and Bob's goal is to prevent it from happening. In 2017, Tuza posed a question about the RRG game asking for which values of $k$ Alice wins the RRG game in a simple graph $G$, given a set of consecutive non-negative integer labels $\mathcal{L}$. We partially answer Tuza's question presenting an upper bound on the number of consecutive non-negative integer labels necessary for Alice to win the RRG game on a simple graph $G$, a cycle, and a path graph.
\end{abstract}

2000 AMS Subject Classification: 05C57, $05 C 78$.

Keywords and Phrases: graph labeling, graceful game, maker-breaker game.

This study was financed in part by the Coordenação de Aperfeiçoamento de Pessoal de Nível Superior - Brasil (CAPES) - Finance Code 001, CAPES-PrInt project number 88881.310248/2018-01, CNPq and FAPERJ. 


\section{Introduction}

A simple graph $G$ consists of a nonempty set $V(G)$ of vertices and a set $E(G)$ of unordered pairs of vertices $u, v \in V(G)$, called edges. The vertices $u$ and $v$ are called endpoints of the edge $u v$. All graphs considered in this paper are simple and finite.

A labeling of a graph is an assignment of labels (elements of a set) to the vertex set (vertex labeling) or the edge set (edge labeling) of a graph.

Graph labeling is an area of study in graph theory whose origin goes back to the well-known Four Color Theorem. Indeed, graph coloring can be viewed as a type of graph labeling since it is an assignment of labels (colors) to the elements of a graph subject to certain conditions.

However, since the 1960s, many other graph labeling problems have arisen that use numbers as labels and not colors, whose properties to be satisfied are strictly numerical. The first labelings of this type were investigated by Rosa [6], who introduced four types of graph labelings and proved that if a graph $G$ with $m$ edges has any one of that four labelings, then the complete graph $K_{2 m+1}$ can be decomposed into copies of $G$. The interested reader can consult Gallian's dynamic survey on graph labeling [4], that presents a vast literature on graph labeling problems and open conjectures, as well as algorithmic and complexity results.

Let $G$ be a simple graph, where $|V(G)|=n$ and $|E(G)|=m$, and let $f: V(G) \rightarrow\{0, \ldots, k\}$ be an injective function that induces an injective mapping $\phi: E(G) \rightarrow\{1, \ldots, k\}$ given by $\phi(u v)=|f(u)-f(v)|$, for all $u v \in E(G)$.

By Golomb [5], if $k=m$, then graph $G$ is called graceful and $f$ is called a graceful labeling. When $k \geq m$, the function $f$ is said to be a range-relaxed graceful labeling (RRG labeling). Figure 1 exhibits an RRG labeling of a graph that does not have graceful labeling.

A large number of graphs are classified as graceful. Rosa [6] proved that Eulerian graphs such that $m \equiv 1,2(\bmod 4)$ are not graceful (it is known as parity condition). The gracefulness of classic families of graphs 
like paths, cycles, complete bipartite graphs [6], and many other families of graphs [4] is already established.

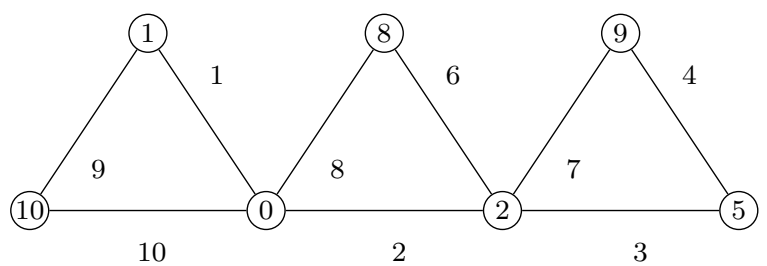

Figure 1: RRG labeling of a graph with no graceful labeling.

In 2017, Tuza [8] surveyed the area of labeling games, proposing the study of new game variants. Combinatorial games are a branch of mathematics that includes no chance games in which players take turns with perfect information until one of them is the winner. It is important to observe that there is absolutely no luck involved and there is no randomness. The range-relaxed graceful game and the graceful game are examples of combinatorial labeling games introduced by Tuza [8].

The range-relaxed graceful game is a maker-breaker game played on a graph $G$ and it is defined as follows: Alice (the maker) and Bob (the breaker) alternately assign an unused label $f(v) \in\{0, \ldots, k\}, k \geq|E(G)|$, to a previously unlabeled vertex $v \in V(G)$. A vertex of $G$ is unlabeled if no label has been assigned to it. If both ends of an edge $u v \in E(G)$ are already labeled, then the (induced) label of the edge is defined by $\phi(u v)=|f(u)-f(v)|$. Note that after any move, the next player can only label unlabeled vertices. A move is legal if, after it, all edge labels are distinct. Only legal moves are allowed during the game and both players play optimally. Alice's goal is to construct a range-relaxed gracefully labeled graph and Bob's goal is to prevent this. The game ends if an RRG labeling is created (Alice wins) or there is no possible legal move (Bob wins). If $k=|E(G)|$, then the game is called graceful game.

Tuza [8] called RRG game as edge-difference distinguishing game. However, we call it a range-relaxed graceful game in order to match with the range-relaxed graceful labeling nomenclature previously established in the 
literature [1, 2, 7].

The graceful game was later studied by Frickes et al. [3] who investigated winning strategies for Alice and Bob in some classic families of graphs such as paths, complete graphs, cycles, complete bipartite graphs, caterpillars, trees, gear graphs, helms, web graphs, prisms, hypercubes, and 2-powers of paths.

In this work, we investigate the RRG game, presenting an upper bound on the number of consecutive non-negative integer labels necessary for Alice to win the RRG game on a simple graph $G$ and contribute to the study of the question posed by Tuza [1]:

Question 1.1 (Tuza [8]). Given a simple graph $G$ and a set of consecutive non-negative integer labels $\mathcal{L}=\{0, \ldots, k\}$, for which values of $k$ Alice wins the range-relaxed graceful game?

In the following section, we present definitions that are useful in this work and show our main results on the RRG game.

\section{Main results}

Before showing our results, first, we present notations and definitions used throughout the text. Let $G=(V(G), E(G))$ be a graph. Two vertices $u, v \in V(G)$ are adjacent if $u v \in E(G)$; in such a case, edge $e=u v$ and vertices $u$ and $v$ are called incident, and vertices $u$ and $v$ are also called neighbors. Note that $u v=v u$, for all $u, v \in V(G)$. The set of neighbors of a vertex $v \in V(G)$ is usually denoted by $N(v)$. The degree of a vertex $v \in V(G)$ is the number of edges incident to $v$ and is denoted by $d(v)$. The maximum degree of $G$ is the number $\Delta(G)=\max \{d(v): v \in V(G)\}$.

A path $P_{n}$ is a graph whose vertices can be arranged in a linear sequence $\left(v_{1}, v_{2}, \ldots, v_{n}\right)$ in such a way that two vertices are adjacent if and only if they are consecutive in the sequence. Similarly, a cycle $C_{n}$ on three or more vertices is a graph whose vertices can be arranged in a cyclic sequence $\left(v_{1}, v_{2}, \ldots, v_{n}, v_{1}\right)$ in such a way that two vertices are adjacent if and only if they are consecutive in the sequence. 
Given a graph $G$, its game graceful number, denoted by $\operatorname{grac}_{g}(G)$, is the minimum non-negative integer $k$ such that Alice wins playing the RRG game on $G$ with a set $\mathcal{L}=\{0, \ldots, k\}$. In the context of the RRG game, we say that a vertex label $\ell \in\{0, \ldots, k\}$ is available to a vertex $v$ of a graph $G$ if it was not assigned to any other vertex of $G$ and if it can be assigned to $v$ without generating repeated induced edge labels.

Next, we present the main result of this work: Alice always wins the RRG game on a simple connected graph $G$ (independently of who starts the game) using a set of consecutive integer labels $\mathcal{L}=\{0, \ldots, k\}$ for any $k \geq(n-1)+\frac{\Delta(\Delta-1)}{2}+\max \{d(v) \cdot 2(m-d(v)): v \in V(G)\}$. This result partially answers Question 1.1 posed by Tuza [8].

Theorem 2.1. If $G$ is a simple connected graph on $n$ vertices, $m$ edges and maximum degree $\Delta$, then

$$
\operatorname{grac}_{g}(G) \leq(n-1)+\frac{\Delta(\Delta-1)}{2}+\max \{d(v) \cdot 2(m-d(v)): v \in V(G)\} .
$$

Proof. Let $G$ be a graph as described in the hypothesis. Also, let $\mathcal{L}=$ $\{0, \ldots, k\}$ be a set of consecutive integer labels such that $k \geq(n-1)+$ $\frac{\Delta(\Delta-1)}{2}+\max \{d(v) \cdot 2(m-d(v)): v \in V(G)\}$. Alice (or Bob) starts the range-relaxed graceful game on $G$ and our objective is to show a winning strategy for Alice.

For each vertex $w \in V(G)$, define a set $L(w)$ called the set of available labels for $w$. At the beginning of the game, let $L(w)=\mathcal{L}$ for all $w \in V(G)$. At each round of the game, a player (Alice or Bob) chooses an unlabeled vertex and assigns to it an available label $\alpha$ such that $0 \leq \alpha \leq k$ (recall that a label $\alpha \in\{0, \ldots, k\}$ is available to a vertex $w$ of $G$ if it was not assigned to any other vertex of $G$ and it can be assigned to $w$ without generating repeated induced edge labels). Right after a player's move, the sets of available labels of the remaining unlabeled vertices are updated so as to maintain the property that these sets only contain available labels for the respective vertices. Thus, when it is Alice's turn, she always choose an unlabeled vertex $w$ and assign to $w$ any label in the set of available labels $L(w)$ if $L(w) \neq \emptyset$. At the end of this proof, we show that $L(w) \neq \emptyset$ 
for every unlabeled vertex $w \in V(G)$ at any point of the game. Next, it is described how the sets of available labels of unlabeled vertices are updated right after each player's move.

At the $j$-th move, a player (Alice or Bob) chooses an unlabeled vertex $v_{j} \in V(G)$ and assigns an available label $f\left(v_{j}\right)$ to $v_{j}$. Right after the $j$-th move, the set of available labels $L(u)$ of each remaining unlabeled vertex $u \in V(G)$ is updated. Only unused vertex labels and vertex labels that cannot generate repeated edge labels in future iterations can remain in each set. The sets of available labels are updated according to the following three steps:

1. for every unlabeled vertex $u \in V(G)$, remove $f\left(v_{j}\right)$ from $L(u)$. Note that, since a range-relaxed graceful labeling is injective, the label $f\left(v_{j}\right)$ cannot be assigned to more than one vertex;

2. for every unlabeled vertex $u \in N\left(v_{j}\right)$ and for every labeled vertex $w \in N(u)$, with $w \neq v_{j}$, such that $f(w) \equiv f\left(v_{j}\right)(\bmod 2)$, delete from $L(u)$ the label $\left(f(w)+f\left(v_{j}\right)\right) / 2$. Note that the deletion of label $\left(f(w)+f\left(v_{j}\right)\right) / 2$ from $L(u)$ is necessary since it generates the repeated edge label $\left|f(w)-f\left(v_{j}\right) / 2\right|$ at edges $u v_{j}$ and $u w$;

3. for every unlabeled vertex $u \in V(G)$ and for every labeled vertex $u^{\prime} \in N(u)$, delete from $L(u)$ every label $\ell$ such that $\left|\ell-f\left(u^{\prime}\right)\right|=\phi(e)$, for every edge $e \in E(G)$ that has both endpoints labeled.

Next, we determine the maximum number of labels that are deleted, throughout the game, from each set of available labels. First, note that exactly one label is deleted from each unlabeled vertex at each execution of Step 1. Thus, exactly $n-1$ labels are deleted at Step 1 after the first $n-1$ moves.

Now, we count how many labels are deleted after the first $n-1$ executions of Step 2. All vertices of $G$ have degree at most $\Delta$. Thus, after the first $n-1$ moves, at most $\left(\begin{array}{l}\Delta \\ 2\end{array}\right)=\frac{\Delta(\Delta-1)}{2}$ labels are deleted from the set of available labels of any unlabeled vertex of $G$, including the last unlabeled vertex. The number $\left(\begin{array}{c}\Delta \\ 2\end{array}\right)$ corresponds to the maximum number of distinct 
vertex labels that can be obtained by taking the $\Delta$ labels of the neighbors of $u$ in pairs, summing them up and dividing them by two.

Next, we count how many labels are deleted after the first $n-1$ executions of Step 3. Let $u$ be the last unlabeled vertex. Right after $n-1$ moves, all the neighbors of $u$ are labeled and there are exactly $m-d(u)$ edges in $G$ with induced labels. For each neighbor $w \in N(u)$, each induced edge label $\ell^{\prime}$ can preclude at most two vertex labels from being assigned to vertex $u$, namely, the labels $f(w)+\ell^{\prime}$ and $f(w)-\ell^{\prime}$. Thus, each neighbor of $u$ contributes for the deletion of at most $2(m-d(u))$ labels from $L(u)$. Since $u$ has $d(u)$ neighbors, the number of labels deleted from $L(u)$ after the first $n-1$ executions of Step 3 is at most $d(u) \cdot 2(m-d(u))$. Therefore, the maximum number of labels that can be excluded on Step 3 is $\max \{d(u) \cdot 2(m-d(u)): u \in V(G)\}$.

From the previous analysis, we conclude that at most $(n-1)+\frac{\Delta(\Delta-1)}{2}+$ $\max \{d(v) \cdot 2(m-d(v)): v \in V(G)\}$ labels are deleted from each set of available labels. Since $|\mathcal{L}|$ is greater than this value, we conclude that there is always an available label at each set $L(u)$ to be assigned to an unlabeled vertex $u$, and the result follows.

Frickes et al. [3] proved that Alice wins the graceful game $(k=|E(G)|)$ on the cycle $C_{3}$, but loses on any larger cycle. This implies that $\operatorname{grac}_{g}\left(C_{n}\right) \geq$ $\left|E\left(C_{n}\right)\right|+1=n+1$ for every $n \geq 4$. Since every cycle $C_{n}$ with $n \geq 3$ has maximum degree $\Delta=2$ and has $n$ edges, the next result follows immediately from Theorem 2.1 and from these observations.

Corollary 2.1. For any integer $n \geq 4, n+1 \leq \operatorname{grac}_{g}\left(C_{n}\right) \leq 5 n-8$. Moreover, $\operatorname{grac}_{g}\left(C_{3}\right)=3$.

From Corollary 2.1, we know that there exists at most $5 n-8$ different labels in the set $\mathcal{L}$ that can be assigned to a vertex $v_{i} \in V(G)$ in order to obtain a range-relaxed graceful labeling of the graph. Figure 2 exhibits a sequence of moves for the RRG game in a cycle with $n=5$ vertices.

Frickes et al. [3] also proved that Alice loses the graceful game on any path $P_{n}$ with $n \geq 4$. This implies that $\operatorname{grac}_{g}\left(P_{n}\right) \geq\left|E\left(P_{n}\right)\right|+1=n$ for 
$n \geq 4$. They also proved that, on $P_{3}$, the winner is the player who starts the game; and that Alice wins on $P_{1}$ and $P_{2}$.

A similar result as Corollary 2.1 can be obtained for paths.

Corollary 2.2. For any integer $n \geq 3, n \leq \operatorname{grac}_{g}\left(P_{n}\right) \leq 5 n-12$. Moreover, $\operatorname{grac}_{g}\left(P_{n}\right)=n-1$ for $n \in\{1,2\}$.

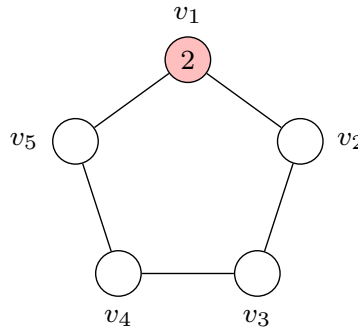

(a) $1^{\text {st }}$ move.

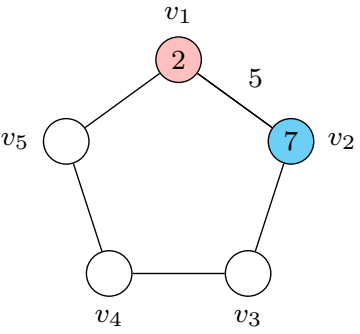

(b) $2^{\text {nd }}$ move.

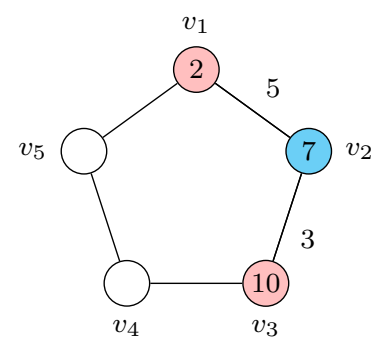

(c) $3^{\text {rd }}$ move.

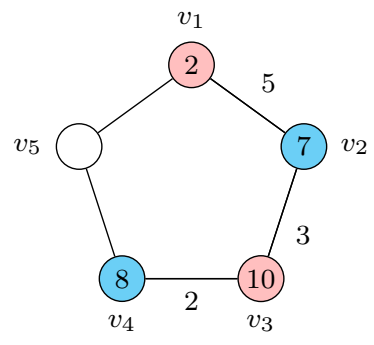

(d) $4^{\text {th }}$ move.

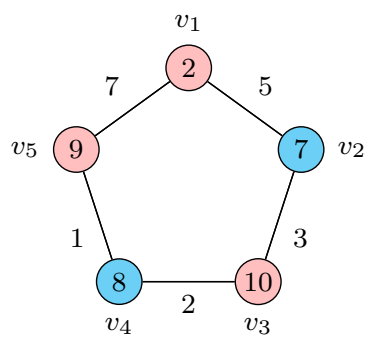

(e) $5^{\text {th }}$ move.

Figure 2: A sequence of moves of the RRG game illustrating the strategy described in the proof of Theorem 2.1. A player chooses an unlabeled vertex $v_{i}$ of the cycle $C_{5}$. At the beginning of the game, $L\left(v_{i}\right)=\mathcal{L}=$ $\{0, \ldots, 17\}$, for every $v_{i} \in V(G)$. After each iteration, the set of available labels is updated for each vertex not labeled. In the last move we guarantee that there exists an available label that can be assigned for the last vertex $v_{i}$ in the set $L\left(v_{i}\right) \subseteq \mathcal{L}$. 


\section{References}

[1] C. Barrientos and E. Kroop, Improved bounds for relaxed graceful trees, Graphs and Combinatorics 33 (2017), 287-305.

[2] F. V. Bussel, Relaxed Graceful labelings of trees, The Eletronic Journal of Combinatorics 9 (2002), 1-12.

[3] L. Frickes, S. Dantas, and A. G. Luiz, The graceful game, In: Proceedings of the 17th Cologne-Twente Workshop on Graphs and Combinatorial Optimization, Enschede, Netherlands (2019), 45-48.

[4] J. A. Gallian, A dynamic survey of graph labeling, The Eletronic Journal of Combinatorics DS6 (2019), 1-535.

[5] S. W. Golomb, Graph Theory and Computing, chapter How to number a graph, Academic Press, New York (1972), 23-37.

[6] A. Rosa, On certain valuations of the vertices of a graph, Theory of Graphs, International Symposium, Rome, July 1966 (1967), 349-355.

[7] G. Sethuraman, P. Ragukumar, and P. J. Slater, Any tree with $m$ edges can be embedded in a graceful tree with less than $4 \mathrm{~m}$ edges and in a graceful planar graph, Discrete Mathematics 340 (2017), 96-106.

[8] Z. Tuza, Graph labeling games, Eletronic Notes in Discrete Mathematics 60 (2017), 61-68. 
Deise L. de Oliveira

Niterói - Brazil

Fluminense Federal University

Federal Institute of Rio de

Janeiro

deiseoliveira@id.uff.br

Atílio G. Luiz

Quixadá - Brazil

Federal University of Ceará

Campus Quixadá

gomes.atilio@ufc.br
Simone Dantas

Niterói - Brazil

Fluminense Federal University

de IME - Campus Gragoatá

sdantas@id.uff.br 